\title{
Perspective actionnelle et internet exemple de projet réalisé en classe d'espagnol en france
}

\author{
LAURE RIPORTELLA \\ MCF IUFM de Lorraine, Nancy, France
}

Received: 9 January 2008 / Accepted: 15 February 2008

ISSN: $1697-7467$

\begin{abstract}
RÉSUMÉ : Le Cadre européen commun de référence pour les langues $(C E C R L)^{1}$ prône une amélioration de la compétence de communication en classe de langue, une approche actionnelle reposant sur la réalisation de tâches communicatives et d'activités de communication langagière par les élèves. Dans ce contexte, les enseignants de langue sont appelés à encourager les projets menés par Internet entre leurs élèves et des élèves natifs. L'auteur de cet article se propose d'amorcer un état des lieux de ces nouvelles pratiques pédagogiques en France à partir d'une enquête menée auprès de plusieurs groupes d'élèves français apprenant l'espagnol comme langue étrangère, il présente la réalisation d'un projet de classes virtuelles mené par un lycée français en classe d'espagnol, et tente de dresser un bilan sur les caractéristiques de ce type de projet, afin de vérifier si ces pratiques innovantes sont en adéquation avec les nouvelles directives en matière d'enseignement/apprentissage des langues et d'en mesurer les apports.
\end{abstract}

Mots-clés : Perspective actionnelle, Internet, tâches communicatives, projet.

RESUMEN : El Marco europeo común de referencia para las lenguas (CECRL), preconiza mejorar la comunicaciün entre los alumnos que aprenden un idioma, practicar una metodologßa accional que proponga realizar tareas y actividades de comunicaciün. Dentro de este marco, se les aconseja a los profesores de idioma valorizar los proyectos que permitan el uso de Internet, entre sus alumnos y alumnos nativos.

El autor de este artículo, se propone iniciar una presentación de la nuevas prácticas pedagógicas en Francia a partir de une investigación dirigida a varios grupos de alumnos franceses que aprenden el español como segundo idioma, presenta la realización de un proyecto de clases virtuales por un instituto francés en clase de español e intenta presentar un balance sobre las características de ese tipo de proyecto con el propósito de averiguar si estas prácticas innovadoras están en correlación con las nuevas directivas del aprendizaje de los idiomas y de evaluar sus aportes.

Palabras claves : Perspectiva accional, Internet, tareas comunicativas, proyecto.

1. Le Cadre européen commun de référence pour les langues est un document élaboré par des experts du Conseil de 1'Europe et qui a été adopté officiellement en 2001. Il sert de référence aux nouveaux programmes des langues de collège et de lycée en France. Il est publié aux éditions Didier (nouvelle édition 2006) et consultable sur Internet. Il s'accompagne du Portfolio européen des langues, qui suit l'élève puis l'étudiant tout au long de son cursus et permet d'évaluer ses niveaux de compétences dans les différentes activités langagières. 
Cet article complète une communication présentée par l'auteur les 26 et 27 avril 2007, lors du colloque de l'Université de Montpellier, intitulé : «La correspondance dans le monde méditerranéen et ses périphéries (XVIe -XXIe siècles). Pratiques sociales et représentations culturelles».

\section{INTRODUCTION : RAPPEL DE QUELQUES CONCEPTS CLÉS DE LA DIDACTIQUE DES LANGUES EN RELATION AVEC NOTRE SUJET}

Traditionnellement, les échanges épistolaires entre correspondants français et étrangers en classe de langue constituaient la seule pratique pédagogique en France qui permettait d'établir un contact, par écrit, avec un natif dont les élèves avaient, en général, obtenu les coordonnées par leur enseignant.

L'intérêt de cette démarche était, pour l'essentiel, d'être capable de réutiliser les éléments linguistiques étudiés en classe pour se présenter physiquement, parler de ses goûts, de ses loisirs, de sa famille. Mais les lettres échangées, qui s'accompagnaient souvent de photos personnelles, voire de cartes postales, avaient également comme objectif de permettre une meilleure connaissance des us et coutumes du pays dont on apprenait la langue, de ses réalités sociales, économiques et culturelles. Ces relations épistolaires permettaient même, dans le meilleur des cas, d'inviter son correspondant et d'être invité par lui, pour un court séjour, dans les familles concernées ; elles s'arrêtaient souvent après un laps de temps, plus ou moins long, simplement par lassitude : un des correspondants tardant trop à répondre, et/ ou par manque de perspective et de projet commun.

Ces pratiques pédagogiques existent toujours. Mais l'existence d'Internet et les multiples possibilités offertes par les nouvelles technologies ont donné un nouvel essor à ces échanges épistolaires en classe de langue. Les nouvelles instructions de l'enseignement des langues et le Cadre européen commun de référence pour les langues, qui privilégient la compétence communicative, devraient également, dans les années à venir, permettre un développement sans précédent de ce type de pratique qui s'intègre parfaitement à la «perspective actionnelle» centrée sur des «tâches» et à la pédagogie de «projet» attendues dans ces nouvelles instructions.

Le lecteur désireux de revoir ces notions pourra se référer aux nombreux articles écrits sur ces sujets par Christian Puren, Professeur des Universités à l'Université de Tallin et à l'Université de Saint Etienne, notamment «Innovation et cohérence en didactique des langues» paru en avril 2002 dans la revue New Standpoints. Dans cet article, Christian Puren, reprend une des définitions de la «tâche» donnée par David Nunan (Nunan, 1989) qu'il nous présente comme «un des didacticiens les plus connus de l'approche par les tâches». Cette définition correspond à ce qui était attendu dans «l'approche communicative» des années 1980-1990 : «Tâche : partie du travail de classe qui fait que les apprenants comprennent, manipulent, produisent et communiquent entre eux dans la langue cible en centrant leur attention sur le sens plus que sur la forme» ( in Puren, 2002:2 ). Et Christian Puren rappelle que «l'attention sur le sens plus que sur la forme » est en effet l'une des orientations majeures de l'approche communicative»; il préfère, quant à lui, donner à cette notion de «tâche» une définition plus abstraite : «unité de sens à l'intérieur du processus d'enseignement/apprentissage» (Puren, 2002 : 3). Dans Se former en didactique des langues, il avait également caractérisé la tâche 
comme «une activité définie par un objectif, un dispositif et des modes d'évaluation» (Puren, $1998: 203)$.

Enfin, Le Cadre européen commun de référence pour les langues, propose, lui, la définition suivante de la tâche :

Est définie comme tâche toute visée actionnelle que l'acteur se représente comme devant parvenir à un résultat donné en fonction d'un problème à résoudre, d'une obligation à remplir, d'un but qu'on s'est fixé. Il peut s'agir tout aussi bien, suivant cette définition, de déplacer une armoire, d'écrire un livre, d'emporter la décision dans la négociation d'un contrat, de faire une partie de cartes, de commander un repas dans un restaurant, de traduire un texte en langue étrangère ou de préparer en groupe un journal de classe ». (CECR, $2006: 16)$.

La notion de «tâche» implique donc une «visée actionnelle» et nous faisons à nouveau référence à Christian Puren afin de caractériser cette perspective actionnelle

La perspective actionnelle correspond à la prise en compte d'un nouvel objectif social lié aux progrès de l'intégration européenne, celui de préparer les apprenants à travailler, dans leur propre pays ou dans un pays étranger, avec des natifs de différentes langues-cultures étrangères (comme c'est déjà le cas par exemple d'entreprises en Allemagne où des Allemands, des Espagnols, des Français travaillent ensemble en anglais). Il ne s'agit plus de communiquer avec l'autre (de s'informer et d'informer) mais d'agir avec l'autre en langue étrangère. (Puren, $2002: 3$ ).

Cette perspective actionnelle implique non seulement une entrée par les tâches, mais aussi la réalisation de «projets», et, à ce sujet, nous renvoyons le lecteur au tableau de Christian Puren résumant «l'évolution historique des modes d'entrée en cohérence en didactique scolaire des langues étrangères en France» (Puren, 2002 : 5), ainsi qu'à sa définition du «projet» : «En pédagogie, le projet est un ensemble de tâches impliquant très fortement un collectif d'élèves en vue de la réalisation d'un produit déterminé sur une durée relativement longue (quelques semaines, quelques mois, voire une année entière)» (Puren, 1998, Glossaire). Et c'est cette perspective qui oriente désormais les programmes de l'enseignement/apprentissage des langues en France.

Dans le présent article, nous analyserons d'abord les caractéristiques de ces nouvelles instructions officielles et leurs répercussions sur les pratiques de classe, en ce qui concerne l'usage d'Internet : perspective actionnelle et internet. Puis nous amorcerons un état des lieux sur la correspondance par Internet en classe de langue, et plus particulièrement en classe d'espagnol, grâce à un questionnaire donné aux élèves de plusieurs académies françaises ; enfin nous tenterons de définir et de mesurer les apports de ces échanges par Internet en relation avec les attentes de la perspective actionnelle à partir d'un projet réalisé en classe d'espagnol dans un lycée français. 


\section{INTERNET ET LE CADRE EUROPÉEN COMMUN DE RÉFÉRENCE POUR LES LANGUES $(C E C R L)$}

Dans les objectifs actuels de l'enseignement des langues, envisager un voyage ou un échange scolaire n'est plus une perspective suffisante : il s'agit, à présent, de prévoir la possibilité pour les élèves de devoir un jour travailler dans un autre pays européen. La langue, après avoir été un moyen d'accéder à la culture d'un pays - comme c'était surtout le cas dans l'enseignement de l'espagnol -, est devenue un outil de communication, et elle doit également permettre à présent aux futurs citoyens d'agir et d'interagir sur le monde du travail.

Dans Les Outils du Conseil de l'Europe en classe de langue, Francis Goullier ${ }^{2}$, Inspecteur Général de l'Éducation Nationale en France, rappelle que «le CECR développe une approche actionnelle qui fait reposer l'enseignement et l'apprentissage des langues sur la réalisation de tâches communicatives et sur les activités de communication langagières» (Goullier, 2005 : $6)$.

[...] Aux «activités langagières essentielles qui sont :

- la compréhension de l'oral ;

- la compréhension de l'écrit ;

- l'expression orale en interaction (en situation de dialogue) ;

- l'expression orale en continu (description, exposé, récit, ...) ;

- l'expression écrite (compte rendu, article, ...) ;

le CECRL ajoute :

- l'expression écrite en interaction (échange par courrier électronique, ...)» (Goullier, $2005: 18)$.

Et cette expression écrite en interaction, impliquant l'utilisation d'Internet, est effectivement évoquée dans les nouveaux programmes de collège et de lycée, où l'on trouve par exemple, dans la rubrique «correspondance» : «écrire une carte postale, un message électronique, un SMS» (page 8, programmes des collèges, langues vivantes, espagnol, B.O. $\mathrm{n}^{\circ} 6 \mathrm{du} 25$ août 2005, Hors-série) : en effet, ces tâches sont susceptibles de provoquer une réponse du destinataire et donc d'instaurer une interaction. Bien d'autres possibilités pédagogiques existent, bien sûr, puisque la correspondance est effectivement possible de façon interactive en direct (chat, webcam, visioconférences...) ou en différé (par courriel, blog) et qu'elle peut associer à la fois l'oral, l'écrit, le visuel et les sons.

Sur ce sujet des échanges par Internet en classe d'espagnol, nous donnerons l'exemple d'une approche actionnelle particulièrement intéressante, mais nous voudrions d'abord interroger les pratiques de classe actuelles, voir si les enseignants de langue et singulièrement d'espagnol prennent en compte ces consignes ministérielles et européennes et nous interroger sur l'intérêt réel de ces pratiques pour l'enseignement/apprentissage de la langue.

2. Inspecteur Général de 1'Education Nationale du Groupe des Langues Vivantes, Représentant national et expert auprès de la Division des Politiques Linguistiques du Conseil de l'Europe, Président du Comité européen des langues. 


\section{LA CORRESPONDANCE PAR INTERNET ET LES PRATIQUeS DE ClasSE, APPORTS LINGUISTIQUES, CULTURELS ET COMMUNICATIONNELS}

\subsection{La correspondance par Internet et les pratiques de classe}

Afin de mieux connaître l'importance et le rôle de la correspondance par Internet dans les pratiques de classe, nous avons réalisé, en 2006-2007, une enquête à l'aide d'un questionnaire rempli en classe, de façon anonyme. Ce questionnaire a été rempli par 230 élèves de collège et de lycée, à nombre égal, qui apprennent l'espagnol dans les académies de Grenoble et de Nancy-Metz.

Il est évident que le nombre d'académies contactées et le nombre de classes ayant répondu à notre questionnaire ne nous permettent pas de tirer des conclusions nationales, mais elles nous semblent assez représentatives de la réalité des classes. Les classes interrogées couvrent un éventail social très large, certaines se situant dans des zones classées ZEP (Zone d'éducation prioritaire), d'autres accueillant des élèves de milieux sociaux plus favorisés. Une classe de $2^{\text {e, et } u n e ~ d e ~} 1^{\text {èr }}$ de section européenne sont également concernées.

Ce questionnaire est le suivant :

ENQUÊTE $^{3}$

Cette enquête s'adresse aux élèves de collège et de lycée qui apprennent l'espagnol. Elle est anonyme ; les élèves doivent seulement préciser le niveau de leur classe.

I. J'ai déjà réalisé une correspondance par Internet avec un ou des élèves espagnols : - jamais - rarement - souvent (préciser le nombre de fois et à quelle(s) occasion(s))

II. Dans ce type de correspondance, j'ai déjà utilisé :

le chat $^{4}$, - souvent - rarement - jamais

la visio-conférence,$^{5}$ - souvent - rarement - jamais

une webcam, - souvent - rarement - jamais

III. J'aime (ou j'aimerais) correspondre avec un élève natif (espagnol) par Internet :

- Oui (pour des raisons personnelles ou à l'occasion d'un projet scolaire, préciser)

- Non (pourquoi ?)

3. Les établissements concernés sont : Le collège Haut de Penoy, le collège Montplaisir, le collège Jacques Monod, le lycée Callot, à Nancy (54), le lycée Luis Majorelle à Toul (54), le lycée L'Oiselet à Bourgoin-Jallieu (38).

4. «Parfois écrit «tchat» ou même «tchache», (en anglais : bavarder) le terme officiellement retenu en France - mais très peu utilisé, sans doute à cause de sa connotation surannée - est «causette» (JO du 16/3/1999). Glossaire de François Mangenot, in Le français dans le monde, numéro 40, «Les échanges en ligne dans l'apprentissage et la formation», CLE international, juillet 2006.

5. «Vidéoconférence et visioconférence sont maintenant considérés comme équivalents [...] les outils gratuits de messagerie instantanée comme Skype, Yahoo Messenger, MSN Messenger permettent les échanges un à un en visioconférence si l'on possède une Webcam et une connexion rapide.». Idem. 
IV. Sujets dont j'aime (ou j'aimerais) parler avec un locuteur natif dans ce type de correspondance :

- La musique, (exemples)

- Le cinéma, la télévision (exemples)

- Mes lectures (exemples)

- Ma vie personnelle et familiale (exemples facultatifs)

- Autres (exemples)

V. Dans ce type de correspondance, j'utilise (ou j'utiliserais) :

- La langue espagnole en essayant de faire des phrases complètes et bien construites

- La langue espagnole mais avec des mots et des structures en abrégé comme dans la rédaction des SMS

- La langue espagnole en utilisant un modèle de lettre comme je l'ai appris en classe

- La langue française ou l'anglais ou l'alternance du français et de l'espagnol selon mes possibilités

VI. Afin de personnaliser ma correspondance, j'utilise (ou j'utiliserais) pour accompagner mes messages :

- Des images ou des photos personnelles ou choisies sur un site Internet

- Oui

- Non Pourquoi ?

- D’autres éléments graphiques ou audio : (exemples), pourquoi ?

Questionnaire réalisé par Laure Riportella, MCF IUFM de Lorraine, Nancy, 2006-2007.

Après examen des réponses obtenues, on peut tout d'abord constater que seulement environ $26 \%$ des élèves ont eu l'occasion de réaliser une correspondance par Internet (20\% rarement et 6\% souvent). (Question I). Et cette réalité ne semble pas concerner seulement les cours d'espagnol, puisque, comme le souligne un article de Christian Bonrepaux dans Le Monde de l'éducation de janvier 2007 «les enseignants sont encore peu nombreux aujourd'hui à utiliser les nouvelles technologies face à leurs élèves. Manque d'équipement ? Pas vraiment : la distribution massive d'ordinateurs portables au collège dans les départements des Bouchesdu-Rhône, des Landes et d'Ille-et-Vilaine n'a pas entraîné un développement notable des usages $^{6}$. La formation est au cœur du problème [...]». (Bonrepaux, $2007: 60$ ).

On comprend donc que le cahier des charges de la formation des maîtres en Institut Universitaire de Formation des Maîtres, qui est paru au B.O. $\mathrm{n}^{\circ} 1$ du 4 janvier 2007, prévoie, dans les dix compétences attendues du futur enseignant, la maîtrise des technologies de

6. Un rapport établi par l'Inspection générale des finances, le Conseil général des technologies de l'information, l'Inspection générale de l'Éducation nationale et l'Inspection générale de l'administration de l'Éducation nationale et de la recherche montre que l'équipement disponible en France est supérieur à la moyenne européenne : 8 élèves par ordinateur contre 8,8 en moyenne européenne. (Site de 1'APLV, Les Langues Modernes) 
l'information et de la communication. Et un certificat évaluant le niveau de compétences informatiques acquises ainsi que les compétences quant aux applications pédagogiques des TICE dans les matières respectives sera délivré à l'issue de la formation : le C2I niveau 2. En effet, le brevet d'enseignement informatique et Internet (B2I) sera pris en compte dès la session 2008 du diplôme national du brevet, les élèves devront obtenir le B2I en lycée et les étudiants le C2I à l'université. Ceci implique que cette formation devra également être réalisée en formation continue pour les enseignants qui n'auront pas bénéficié de la formation initiale en IUFM et une plate-forme Web nationale est envisagée dans ce but par le ministère de l'Education Nationale. (Bonrepaux, 2007 : 61).

Ce manque de formation des enseignants concerne la maîtrise des outils informatiques, mais aussi les possibilités d'utilisation de ces outils sur le plan de la pédagogie de la discipline. Il faut donc espérer que les mesures ministérielles de formation des maîtres se révèleront efficaces et qu'elles auront comme conséquence de permettre très rapidement aux élèves d'utiliser l'outil informatique en classe de langue. En effet, la demande d'échanges interactifs par le biais d'internet est certes institutionnelle, mais elle est également liée à des pratiques de plus en plus ancrées dans le quotidien des élèves.

C'est sans doute ce qui explique que plus de $75 \%$ des élèves consultés souhaiteraient être initiés à ce genre de pratique (Question III). Les arguments évoqués sont, dans l'ordre de priorité : améliorer la compréhension et l'expression de la langue orale et écrite, connaître des expressions typiquement espagnoles, envisager un projet scolaire, connaître la culture du pays, développer le côté ludique de l'enseignement/apprentissage de la langue, se faire des amis espagnols parce qu'on aime bien les Espagnols, prévoir de travailler un jour en Espagne (1 élève).

On note, tout de même, qu'environ $22 \%$ des élèves interrogés ne souhaitent pas correspondre par Internet avec un natif ; on trouve la majorité d'entre eux en collège et leurs arguments sont les suivants : «parce que je n'ai pas trop le temps, que j'ai trop de devoirs et que c'est trop compliqué», «parce que ça ne remplace pas un vrai contact entre deux personnes», "ça ne m'intéresse pas et les voyages en Espagne ne se font jamais», "parce que je ne vais rien comprendre et que je ne veux pas chercher ce qu'ils disent», «parce qu'ils écrivent en abrégé» ( 1 élève), «parce que je ne fais pas trop confiance aux autres sur Internet quand je ne les connais pas» (1 élève), «parce que je n'aime pas la langue espagnole» ( 1 élève), «parce que je n'ai pas d'ordinateur» (1 élève)».

\section{2 Échanges par Internet : apports linguistiques, culturels et communicationnels}

On constate donc que l'objectif de correction et d'enrichissement de la langue est largement prioritaire dans les réponses des élèves. D'ailleurs, les réponses à la question numéro $\mathbf{V}$ confirment cette tendance puisque la majorité d'entre eux (50\%) essaie ou essaierait d'utiliser des phrases complètes et bien construites dans ce type de correspondance, et $17 \%$ un modèle de lettre comme cela est étudié en classe. Le fait que ce questionnaire ait souvent été rempli en cours a peut-être influencé les réponses, même si celles-ci étaient anonymes. Mais nous nous interdisons ici de faire des procès d'intention aux élèves.

D'ailleurs, environ $14 \%$ des élèves envisagent d'utiliser également des mots et des structures en abrégé comme dans les SMS et environ 40\% d'utiliser également la langue française ou anglaise ou l'alternance du français et de l'espagnol selon leurs possibilités (plusieurs cases pouvaient être cochées). 
De fait, si l'on s'intéresse aux apports linguistiques des correspondances par Internet, les études menées en français langue étrangère et dans les autres langues sur les productions par ordinateur donnent des réponses assez contradictoires. En ce qui concerne l'écriture synchrone, avec réponses immédiates, Richard Kern a conclu que ce type d'activité stimule:

l'expression libre des idées, la motivation et l'initiative dans la communication, l'énonciation des différences d'opinion, la multiplication des perspectives sur les sujets discutés et la réduction des différences de statut et de pouvoir parmi les participants (professeurs et élèves). En revanche, la CMO (communication médiatisée par ordinateur) synchrone n'était pas favorable au renforcement des normes, qu'il s'agisse de perfectionnement grammatical et stylistique, de cohérence globale ou de consensus. (Kern, $1995: 18$ ).

En effet, le débit rapide de la communication en face à face entraîne des erreurs morphosyntaxiques et ne permet pas un temps de correction.

Par contre, nous dit Richard Kern, Madame Pellettieri, qui a étudié le discours de dix binômes d'étudiants d'espagnol qui utilisaient le logiciel synchrone e-talk, et qui a également analysé les modifications faites par les étudiants en réponse à ce que l'on appelle le feedback ${ }^{7}$ (questions, demandes de clarification, corrections, etc.), a trouvé que :

les étudiants incorporaient jusqu'à $75 \%$ du feedback dans leurs productions suivantes et [qu]'ils se corrigeaient presque toujours dans la direction de la norme grammaticale. Donc, au contraire de Kern, Pelletieri a conclu que la CMO synchrone contribuait au développement de la compétence grammaticale et à la conscience métalinguististique des apprenants. (Kern, 1995 : 19).

Les avis sont donc partagés à ce sujet, mais il ne fait pas de doute que la tâche pédagogique définie par l'enseignant, les consignes données par celui-ci et une éventuelle évaluation de cette correspondance peuvent influencer les acquis linguistiques obtenus. Le problème qui se pose est de savoir si, dans ce type de démarche, on privilégie la spontanéité de la communication ou les apports linguistiques. En effet, comme nous l'avons vu en introduction à cet article, la définition de» la tâche» donnée par David Nunan met l'accent, dans ce type de communication, «sur le sens plus que sur la forme» (Nunan, 1989, in Puren, 2001 : 2).

La question IV du questionnaire s'intéressait aux thèmes que les élèves aimeraient aborder avec leurs correspondants lors d'une correspondance La musique (les groupes qu'ils écoutent, le hard rock, le rap, la techno, le reggae) avec 69\% des réponses, la télévision (avec les séries télévisées) et le cinéma (les films d'action, d'horreur) avec 58\% viennent nettement en tête, puis suivent la vie personnelle et le cadre de vie (présenter sa ville) avec $30 \%$; les lectures arrivent en quatrième position avec $22,5 \%$, enfin, dans «autres thèmes», le sport obtient $10 \%$, suivi de près par les jeux vidéo.

7. Les termes anglais semblent incontournables dès qu'il s'agit d'Internet. 
En ce qui concerne les lectures, quelques élèves ont même précisé «je ne lis pas». Ceux qui souhaitent parler de leurs lectures sont très majoritairement en lycée (ils citent : BD, livres sur les vampires ou sur le rap), un élève de $1^{\text {ère }} \mathrm{L}$ donne comme exemples de lectures dont il aimerait parler à son correspondant Sauve-moi de Guillaume Husso et L'Enfant de Jules Vallès ; un autre élève souhaiterait connaître les œuvres espagnoles et aimerait que cette correspondance par Internet permette de se conseiller mutuellement des lectures, enfin un élève aimerait échanger des avis sur des œuvres de théâtre et notamment ... de Shakespeare.

Fait surprenant, un seul élève de TL a coché la rubrique «lecture» dans le questionnaire: cet élève souhaiterait connaître les œuvres étudiées en classe par son correspondant.

On peut s'interroger sur le manque d'intérêt suscité par la lecture dans une éventuelle correspondance par Internet et sur le fait que même des élèves de TL soient si peu motivés pour échanger des avis dans ce domaine. On pourrait suggérer que les élèves qui choisissent la filière L ne sont pas (ou plus) forcément de grands lecteurs, notamment d'œuvres littéraires, mais on peut également émettre d'autres hypothèses. Les élèves, on le sait (Riportella, 2006: 20), établissent une différence entre les lectures personnelles considérées comme ludiques, les exemples donnés sont la $\mathrm{BD}$, les magazines, les romans policiers ou de science-fiction, et celles qui sont étudiées en classe et donc considérées comme un exercice scolaire qui apporte des connaissances, mais qui n'a pas vraiment d'incidence sur leur vie personnelle. On ne peut, certes, que regretter cet état de fait, comme le souligne d'ailleurs Todorov dans son livre Littérature en péril (Todorov, 2007) et espérer que de nouvelles approches du texte littéraire permettront un jour de rendre ces textes plus familiers aux élèves ${ }^{8}$.

Enfin, toujours dans cette question IV, un ou une élève souhaite parler du système scolaire espagnol dans la rubrique «autres sujets».

L'aspect culturel n'est donc pas absent de ce que pourrait être ce type de correspondance par Internet, mais la vie quotidienne et personnelle est nettement privilégiée, ainsi que le côté ludique des échanges.

Par ailleurs, les élèves sont également demandeurs d'une meilleure maîtrise des outils informatiques puisque, à la question VI, ils sont $62 \%$ à répondre qu'ils souhaiteraient pouvoir intégrer à leur correspondance des photos personnelles, de leur famille, de leur ville et de leur région, les musiques qu'ils aiment, afin de mieux se connaître et se comprendre car, dit un élève «quand les mots passent difficilement, les images peuvent faire le reste», parce que «les images donnent plus envie de lire un texte», et parce que «images et musiques permettent de mieux illustrer les sentiments», "parce que c'est plus beau», " para que sea más divertido» a répondu un élève, «parce que c'est plus personnel» ont répondu certains.

Là encore, on peut noter qu'images et musiques semblent plus efficaces que l'écriture pour rendre compte des aspects les plus personnels des individus. Voilà qui pourrait, certes, confirmer les constats actuels sur l'importance des images et des sons dans notre société, mais qui met peut-être aussi en évidence une lacune de notre système scolaire : les travaux d'écriture, pourtant exigés au baccalauréat de français, et l'expression écrite, activité langagière faisant partie de la compétence de communication en langue, ne semblent pas, dans la pratique quotidienne, familiariser suffisamment les élèves avec l'écrit. Une fois de plus, les travaux

8. Si nous insistons sur ce sujet, c'est parce que le texte littéraire est, traditionnellement, un support privilégié dans l'enseignement:/apprentissage de l'espagnol en France. (Riportella, 2006). 
scolaires et la vie personnelle ne semblent pas se rejoindre. Même si, bien entendu, il serait absurde de ne pas utiliser les avantages que peuvent procurer les nouvelles technologies pour enrichir les possibilités de communication de la correspondance traditionnelle, il ne faudrait pas, non plus, que celles-ci se substituent totalement à l'écriture.

\section{4. ÉCHANGes PAR INTERnet et PERSPECTIVE ACTIONNELle : LA MiSe EN PLA- CE DE PROJETS ENTRE CLASSES VIRTUELLES}

Afin d'illustrer notre propos nous allons présenter le projet qui a été réalisé par des professeurs d'espagnol du lycée l'Oiselet de Bourgoin-Jallieu (38), lycée qui a également participé à notre enquête ${ }^{9}$ et par leurs élèves. Il faut préciser que les autres langues représentées dans cet établissement (allemand, anglais, italien) sont également concernées par ce projet, qui nous semble correspondre exactement aux attentes du Cadre européen commun de référence pour les langues, même si les correspondants ne sont pas, dans ce cas précis, des natifs, mais des lycéens de différents pays qui apprennent la langue espagnole au lycée. D'où la création de classes virtuelles composées de groupes de deux ou trois adolescents de 15 à 18 ans, qui vont correspondre avec d'autres groupes de pays étrangers (Italie, Etats-Unis...) et participer, avec leur professeur d'espagnol, à un projet d'échange culturel intitulé cette année 20062007: "Travailler ensemble sur le thème de la consommation des jeunes- Travailler ensemble sur le thème du commerce équitable».

Ce projet, que l'on peut qualifier de culturel, d'actionnel et de communicationnel notions que nous avons abordées en introduction à cet article et lors de l'analyse des résultats du sondage - est constitué de trois parties :

a. apprendre à se connaître

b.travailler ensemble sur le thème de la consommation chez les jeunes

c. travailler ensemble sur le thème du commerce équitable

Les deux premières parties se divisent en plusieurs étapes au cours desquelles les élèves sont incités, à tout moment, à exprimer leurs sentiments, leurs observations et à faire des propositions afin de créer (je traduis): « un espace où la communication et l'échange culturel occupent une place privilégiée», puisqu'il s'agit «de créer des affinités avec ses camarades et de se connaître mieux $»^{10}$. Cela à condition de respecter un «code de bonne conduite» ou "Carta magna», ${ }^{11}$ que les élèves doivent s'engager à accepter, dès septembre, avant la mise en place du projet.

\section{CARTA MAGNA}

Antes de inscribirte con tu nombre de usuario y contraseña, tienes que leer y aceptar las siguientes condiciones :

9. Mes remerciements à mes collègues d'espagnol qui ont accepté de me présenter leur projet.

10. http://wkto.free.fr/as/0607 SCHOOLYEAR/wktoES08.htm

11. Ce projet concerne des élèves qui apprennent l'espagnol, nous publions donc ce «code de bonne conduite» en espagnol, tel qu'il est découvert par les élèves. 
a los alumnos que forman parte de este proyecto cumplan las reglas. Este proyecto, destinado a gente de países tan diferentes, permite conocerse, aprender a comprenderse, a respetarse, a intercambiar ideas sobre temas culturales, literarios, económicos... Por estas razones pedimos tanto a los profesores como siguientes :

1. Lee el proyecto de tu clase virtual: http://wkto.free.fr/as/wktoES15.htm.

2. Sólo incluye fotos, dibujos y texto en tu clase virtual.

3. Está prohibido incluir cualquier material indecente.

4. Respeta las leyes de tu país. En Francia puedes publicar la foto de alguien en Internet sólo con su consentimiento por escrito. Si esta persona es menor de 18 años, debes tener el consentimiento escrito de sus padres.

5. No tipees tu apellido, sólo tu nombre.

6. No debes dar a nadie tu código de acceso ni tampoco lo guardes en el ordenador.

7. Los profesores deben traducir el reglamento a la lengua materna de los estudiantes, así como también, deben supervisar la actividad de sus estudiantes en el entorno digital de Wkto. Los estudiantes no están autorizados a abrir discusiones sobre temas ilegales. Con su código, los profesores pueden abrir y modificar los textos en las diferentes clases virtuales. Los profesores tienen el rol de moderadores: intervienen para modificar los textos escritos por los estudiantes así que los estudiantes deben prestar conformidad al reglamento de buena conducta.

Me comprometo a respetar el reglamento de buena conducta arriba mencionado:

\begin{tabular}{|l|l|l|}
\hline NOMBRE & INSTITUTO DE ENSEÑANZA SECUNDARIA & FIRMA \\
\hline & & \\
\hline
\end{tabular}

On le voit, cette charte pose très clairement les objectifs, les possibilités offertes, mais aussi les limites éthiques. On note que ces consignes sont rédigées en espagnol (ce qui nous renvoie à notre souci concernant les apports linguistiques de ce type de correspondance), et les rôles de correcteur et de modérateur du professeur bien précisés. Cependant, chaque élève dispose de suffisamment de liberté pour laisser s'exprimer sa personnalité.

Lors de la première étape, en novembre, les élèves doivent se présenter par écrit, en espagnol (environ 100 mots), à leur correspondant étranger (portrait physique, loisirs, goûts, traits de caractère), ils peuvent joindre une photo d'eux à ce courrier.

L'étape numéro 2 consiste en une présentation de l'environnement de l'élève (environ 120 mots) : avec une description de cet environnement et une partie plus personnelle dans laquelle l'élève dira comment il vit dans cet environnement et comment il le ressent.

Puis, lors de la troisième étape, en décembre, il s'agira de traduire un questionnaire, élaboré par les professeurs, portant sur la consommation des jeunes afin de connaître les produits achetés prioritairement par les jeunes et leurs critères de choix. Chaque élève fera remplir ce questionnaire, identique, dans son pays, à plusieurs jeunes lors de la quatrième étape. 
Ensuite, les résultats de l'enquête sont envoyés à tous les participants, sous forme de graphique, ou de pourcentages, et chacun peut exprimer ses réactions et ses commentaires à propos de ces résultats (étape 5).

Enfin, au cours du deuxième trimestre, les élèves de ces classes virtuelles auront à réaliser plusieurs publicités pour des produits du commerce équitable qu'ils souhaiteraient créer. Ce projet est mis en place à partir de plusieurs étapes : choix des produits, caractéristiques de ceux-ci, production de la publicité avec rédaction des textes et création des éléments visuels. Chacun fait des propositions et l'on décide ensemble des éléments qui seront retenus. Les productions réalisées sont ensuite exposées dans les Centres de Documentation des différents établissements. Ce travail terminé, les élèves se disent au revoir et mettent un terme aux échanges des classes virtuelles. Entretemps, certaines correspondances personnelles se sont mises en place (échange d'adresses électroniques personnelles, invitations) et pourront se poursuivre hors temps scolaire.

\section{Bilan}

Ce projet nous semble très riche à bien des égards. En effet, les apports linguistiques ne font pas de doute : les consignes en espagnol, la lecture des messages envoyés par les correspondants, l'exercice de traduction, le regard du professeur sur ces productions, amènent les élèves à travailler l'activité langagière de compréhension de l'écrit. La rédaction de messages et des résultats de l'enquête font travailler l'expression écrite. Si la perfection linguistique n'est pas la priorité, l'amélioration du niveau de compétence en langue orale et écrite reste bien entendu un objectif commun aux élèves et aux enseignants.

Le travail mené permet de comparer les goûts en matière de consommation des jeunes de différents pays, il constitue un objectif à la fois culturel et pragmatique motivant et il peut être envisagé dans divers domaines (par exemple connaître les goûts des adolescents dans le domaine littéraire ou artistique, ce qui était le thème du projet de l'année précédente pour les élèves de cet établissement dans le cadre de leurs échanges avec des classes virtuelles).

Enfin, comme nous l'avons déjà souligné, le cadre, bien que rigoureux, n'empêche pas une réelle communication impliquant les individus. Il suffit de consulter, par exemple, quelques productions d'élèves pour s'en rendre compte. Les échanges, lors de la première et de la deuxième étape, notamment, (la présentation individuelle et celle de l'environnement), mais aussi lors de l'étape finale (les commentaires sur les sondages réalisés) montrent que le style utilisé par les correspondants est très différent. On retrouve, certes, des constantes : la description physique, celle de l'environnement, etc., mais le vécu exprimé et les modalités d'écriture sont très différents. Un élève, qui ne manque pas d'humour, (ou d'ambition), annonce, par exemple, à son correspondant, lors d'un échange spontané en direct sur internet, que son projet de vie est d'être, un jour, Président de la République ; une élève de 1'ere ES, qui a accepté que 1'on publie sa lettre, a choisi, quant à elle, de se présenter de la façon suivante, lors d'un échange plus élaboré en différé : (nous ne corrigeons pas les erreurs de langue).

Para comenzar, me llamo Coline. Soy francésa y tengo 16 años. Vivo en un pequeño pueblo que se llama Beauvoir de Marc

Si fuera una flor, sería una margarita porque me gusta la simplicidad.

- Si fuera una fruta, sería una manzana, podemos comerla dulce y si no también salada.

- Si fuera una golosina, sería caramelos porque estoy en contra de la violencia 
- Si fuera una verdura, sería una lechuga porque me gusta la frialdad.

- Si fuera un animal, sería un gato porque soy independiente pero me gusta estar en mi casa.

- Si fuera una película, sería «le fabuleux destin d'Amélie Poulain» por su esperanza.

- Si fuera un compositor, sería Tiersen porque su música evoca la vida.

- Si fuera un instrumento musical, sería un piano porque estoy en comunión con él cuando toca.

- Si fuera un color ; importa poco! ;segun mis humores.

- Sin embargo, actualmente es el negro...

- A través de estas comparacíones, espero que conoceis un poco mejor mi vida, quién soy.

- ¡hasta luego! Coline, Bourgoin.»

Ce ne sont que quelques exemples qui témoignent que les échanges par Internet, lorsqu'ils font partie d'un projet scolaire où la perfection linguistique n'est pas l'objectif premier, permettent aux élèves de communiquer tout en exprimant leur personnalité.

La production d'affiches publicitaires, phase plus complexe, est l'aboutissement de ce projet. Elle permet aux élèves d'apprendre à travailler ensemble et de façon progressive. Elle met en jeu plusieurs types d'apprentissage d'ordre cognitif, linguistique, culturel, et permet une prise en compte de la créativité des élèves, tout en les formant aux multiples possibilités offertes par les nouvelles technologies.

C'est sans doute ce qui explique la forte motivation des élèves, ainsi que celle des enseignants à ce projet.

\section{Conclusion}

En conclusion, on peut noter que les projets menés par Internet, bien qu'encore peu développés en classe de langue, devraient connaître un essor important dans les années à venir. Ils correspondent à une demande institutionnelle, mais également à une attente du public scolaire.

Les apports linguistiques, culturels, communicationnels ne font aucun doute, comme le montre la réalisation du projet que nous avons présenté. Plus ludique et plus variée que ne l'était la correspondance scolaire épistolaire traditionnelle, la correspondance par Internet offre de multiples possibilités, comme celle de joindre des extraits de musique, des documents divers, personnels ou récupérés sur Internet. Elle est également plus motivante pour lire et écrire une langue vivante, notamment parce qu'elle est en prise avec les habitudes des adolescents en matière de communication. En effet, si ceux-ci sont peu nombreux à avoir établi des correspondances écrites ou orales dans le domaine scolaire, il en va autrement lorsqu'il s'agit de leur vie quotidienne (échange de SMS, mais aussi création de blogs et interventions sur d'autres blogs : sur 50 élèves sondés, 20 élèves déclarent avoir un ou plusieurs blogs et 37 en consultent). Cette correspondance par internet a comme atout majeur de permettre à la fois une communication en direct, rapide et spontanée, et une communication en différé, potentiellement plus élaborée. Nous avons vu, grâce à la lettre de présentation de Coline, que ce type de correspondance, même dans le cadre d'un projet scolaire bien défini, pouvait permettre aux élèves d'exprimer leur personnalité et de développer des qualités littéraires. 
De plus, la conception de projets à long terme, comme celui que nous avons présenté, (au moins le temps d'une année scolaire) permet une communication entre les élèves plus suivie que les échanges épistolaires traditionnels et plus constructive puisqu'il s'agit, dans cette perspective actionnelle, d'agir ensemble à partir de thèmes communs de société, de mettre en relation des résultats obtenus après une enquête construite ensemble et d'en tirer des conclusions sur les points communs et les différences, enfin de faire connaître ces conclusions à un public par des modalités décidées ensemble.

Les activités langagières travaillées : compréhension de l'oral et de l'écrit, expression orale interactive et expression écrite interactive et en différé permettent une communication spontanée, mais aussi plus élaborée. Tout cela montre l'efficacité de ce type de projet qui implique également que se développent l'autonomie et la créativité des élèves pour mieux se comprendre, et pour agir plus vite et plus efficacement.

Quant aux enseignants, leurs échanges avec des collègues de pays étrangers enseignant la même langue qu'eux s'avèrent très enrichissants, aussi bien sur le plan pédagogique que sur les plans linguistique et culturel. Le travail réalisé avec leurs élèves, mais aussi avec ceux de leurs collègues étrangers, permet d'établir des relations hors frontières particulièrement motivantes et formatrices.

Pour toutes ces raisons, nous pensons que ce type de projet, en accord avec les directives officielles de l'enseignement/apprentissage des langues en France, mais également en accord avec les directives européennes mérite de se développer en classe de langue.

La correspondance par Internet en espagnol et hors contexte scolaire est également en plein essor. Il existe, par exemple, un certain nombre de blogs d'adolescents espagnols ou de langue espagnole sur Internet qui invitent des adolescents du monde entier à correspondre. ${ }^{12}$

Hors du contexte scolaire, les apports linguistiques, culturels, communicationnels sont sans doute d'une tout autre nature que ceux que nous avons observés précédemment, et ils pourraient nous amener à nous poser des questions que nous ne traiterons pas ici. Mais on peut trouver un certain nombre de ces questions, et quelques réponses, dans le livre de Vincent Cespedes : Mot pour mot, kel ortograf pr $2 m 1$ (Flammarion, 2007). Dans cet ouvrage, un des personnages qualifie Internet (et les téléphones portables), de supports «orthographicides», qui conduisent à un «athéisme orthographique», alors que le second personnage défend, lui, l'idée qu'il faut parvenir à réconcilier les cultures de l'oralité et de l'écrit.

Le débat, on s'en doute, est loin d'être clos et il n'est pas sans rapport avec notre sujet puisqu'une des innovations de l'approche actionnelle est de montrer que l'on peut être efficace et communiquer et agir en langue sans que la perfection de la correction linguistique soit visée.

Le projet de classe mené par Internet que nous avons présenté se situe bien dans cette perspective actionnelle puisqu'il privilégie l'action et la communication, mais il tend à montrer que, même si ces objectifs actionnels et communicationnels sont prioritaires, il est également possible de réconcilier ces deux cultures, de l'oralité et de l'écrit, où, traditionnellement, le statut de l'erreur linguistique est différent.

12. Presque $90 \%$ des adolescents espagnols de 12 à 17 ans utilisent Internet et $57 \%$ d'entre eux ont un type d'activité créative sur le réseau. Et en 2005, sur MSN España, on comptait un million de blogs sur 4 millions de lignes ADSL. 


\section{Bibliographie}

Cadre européen commun de référence pour les langues, apprendre, enseigner, évaluer, Division des Politiques Linguistiques Strasbourg, Didier, 2006. www.coe.int/lang.

Bulletin officiel, numéro 6 du 25 août 2005 hors-série, Ministère de l'Éducation Nationale et de la Recherche, France.

New Standpoints, «Innovation et cohérence en didactique des langues», pages 3 à 7, Christian Puren, 2002.

Se former en didactique des langues, Christian Puren, Bertocchini Paola, Costanzo Edwige, Paris, Ellipses, 1998.

Designing Tasks for the communicative Classroom, David Nunan, Cambridge University Press, 1989.

Le français dans le monde, «Les échanges en ligne dans l'apprentissage et la formation», coordonné par Charlotte Dejean-Thircuir et François Mangenot, CLE international, juillet 2006.

Le Monde de l'éducation, janvier 2007, $\mathrm{n}^{\circ}$ 354, «enseignants : se former aux TICE, et vite !», page 61, 62, Christian Bonrepaux.

Les Outils du conseil de l'Europe en classe de langue, Cadre européen commun et portfolios, Francis Goullier, IGN, Didier, Paris 2005.

Cadre commun européen de référence pour les langues, Apprendre, enseigner, évaluer, Division des Politiques Linguistiques Strasbourg, Didier, 2006.

Le texte littéraire en classe d'espagnol, Laure Riportella, INRP, 2006.

La littérature en péril, Tzvetan Todorov, Flammarion, 2007.

Mot pour mot, kel ortograf pr 2m1, Vincent Cespedes, Flammarion, 2007.

\section{Adresses Internet :}

- www.ac-grenoble.fr/wkto

- http://rvr.typepad.com/linotipo/2004/06/estudio_sobre_e.html

- http://wkto.free.fr/as/0607_SCHOOLYEAR/wktoES08.htm

- site Lindipo

- site Red.es 